\title{
CD4+ cells in autoimmune thyroid disease
}

\author{
Szymon Janyga ${ }^{\circledR 1}$, Bogdan Marek ${ }^{11,2}$, Dariusz Kajdaniuk ${ }^{\circledR 1,2}$, Monika Ogrodowczyk-Bobik ${ }^{(1)}$, \\ Agata Urbanek (101, tukasz Bułdak (ํㅜㄹ
}

${ }^{1}$ Department of Endocrinology and Metabolic Diseases, Regional Specialist Hospital No. 3, Rybnik, Poland

${ }^{2}$ Department of Pathophysiology and Endocrinology, Faculty of Medical Sciences in Zabrze, Medical University of Silesia, Katowice, Poland ${ }^{3}$ Department of Internal Medicine and Clinical Pharmacology, Faculty of Medical Sciences in Katowice, Medical University of Silesia, Katowice, Poland

\begin{abstract}
The phenomenon of autoimmunity develops as a result of the triggering factor released by damaged cells. This leads to an infiltration of $\mathrm{CD} 4+$ cells involved in stimulating the effector cells cytotoxicity and stimulating the humoral response. One of the most common autoimmune disorders are autoimmune thyroid diseases, including Hashimoto's thyroiditis and Graves's diseases. Helper T lymphocytes, which are divided into Th1, Th2, Tregs, and the relatively new groups Th17, Th22, and Th9, are involved in the pathogenesis of AITD. CD4+ cell subtypes mature and differentiate by specific transcription factors and in a specific interleukin environment. Not only are Th1 and Th2 cells involved in the development of AITD, but also Th17, Th22, and Th9 lymphocytes and their correlation to Tregs lymphocytes. The plasticity of the CD4+ cells is very important, affecting the balance between these cells, as well the factors modulating their phenotypic variability. Patients with AITD have an increased percentage of Th17, Th22, and Th9 cells as well as defective function of Tregs lymphocytes. The balance between Th17 cells (and also other cytotoxic T cells) and Treg cells is also very important. Understanding the role of CD4 cells in the pathogenesis of AITD may be important not only for the development of the knowledge, but also for determining therapeutic targets. (Endokrynol Pol 2021; 72 (5): 572-583)
\end{abstract}

Key words: CD4+ T cell; Th lymphocytes; Treg lymphocytes; autoimmune thyroid disease

\section{Introduction}

In recent years, the hypothesis regarding the initiation triggers, the initiation itself, and the course of the autoimmunity process has changed. From Burnet's self-non-self theory [1], through the infection non-self theory by Janeway [2,3] (according to which the antigen-presenting cells [APSs] react with the pathogen-associated molecular patterns [PAMPs], e.g. liposaccharide [LPS] and only their presence initiates the immune response), to Polly Matzinger's danger theory [4]. According to the latter theory, an immune response will be induced only if there is an alarm signal released by the endogenous cells that are damaged (danger/damage-associated molecular patterns [DAMPs]), so the response is a consequence of the appearance of a sent danger signal, whether it comes from the cell itself or from a foreign cell [5]. Among the growing group of already known danger signals (DAMPs) the following should be noted: extracellular nucleic acids, ATP, heat shock proteins, uric acid, and HMGB1 protein, among others.

Thyroid follicular cells have Toll-like receptors (TLRs) that respond to PAMPs and DAMPs. These trig- gers activate the immune response: CD4+ cells influx, secretion of proinflammatory cytokines, and ultimately cell damage and apoptosis [6].

Autoimmune thyroid diseases (AITDs) — of which the main representatives are chronic lymphocytic thyroiditis (Hashimoto's thyroiditis, HT) and Graves's disease (GD) - are the organ-specific autoimmune diseases characterized by a breakdown of self-tolerance to thyroid antigens [7]. Although the AITDs are the most common autoimmune diseases, we still do not know enough about the triggers of autoimmune responses to thyroid antigens: thyroglobulin (Tg), thyroid peroxidase (TPO), and thyrotropin receptor (TSHR). There is widespread consensus that, for the occurrence of the thyroid cell self-destruction, there must be a coincidence of genetic predisposition and an environmental factor. Many genes, including TSHR, TG, HLA-DR3, CTLA-4, and PTPN22, contribute to the predisposition of AITD [8-10].

In recent years, our knowledge about the autoimmune process has expanded significantly. From the basic information on Tc, Th1, and Th2 lymphocytes involved in the destruction or stimulation of the thyroid gland, we know much more about the autoimmune 
response in the thyroid gland. The initial Th1/Th2 paradigm in the AITD process has been changed to the balance and interaction of the relatively new Th17 and Treg lymphocytes, as well as Th22 and Tfh [9]. In order to learn about the importance and interdependence of particular subtypes of CD4+ cells (including Th1, Th2, Th17, and Treg lymphocytes) in the pathogenesis of AITD, we would like to present the current knowledge on their differentiation, division into subtypes, and characteristic functions.

\section{Subtypes and differentiation of the CD4+T cells}

The CD4+ cells are created in the thymus from the naïve CD4+ cells and then appear in the peripheral lymphoid tissues as mature naïve $T$ cells [11]. These cells, with the participation of distinct cytokines and transcription factors, are differentiated into particular $\mathrm{CD} 4+\mathrm{T}$ cell subtypes [12]. Obviously, the process of differentiating mature naïve CD4+ cells into subtypes of CD4+Th cells is preceded by activation of 2 (at least) or 3 (preferably) signals. The first signal is derived from antigen-presenting cells, which present antigen-derived peptides in the context of the major histocompatibility complex MHC class II molecules to the T-cell receptor (TCR) [13]. The second signal is the result of combining co-stimulating molecules. The cytokine receptor stimulation makes the third signal $[13,14]$.

The regulation of the formation and differentiation of individual CD4+ cell subtypes participate in the noncoding RNA chains (ncRNAs), whose disturbed expression is closely related to the pathogenesis of AITD [15].

What is very important (but is not our focus) is the fact that in the thymus, $\mathrm{T}$ lymphocytes mature under a multistage process of positive selection (in the thymic cortex) and negative selection (through the epithelial cells in the medulla of thymus) [9]. In this process some of the leaving the thymus lymphocytes react through the activation initiated by self-antigens; it is one of the triggers of autoimmunity.

The resulting CD4+ cells divide into helper T lymphocytes (Th) and regulatory lymphocytes (Treg).

\section{Th cell subtypes}

The main surface molecule of Th cells that identifies them is CD4 (cluster of differentiation 4). This glycoprotein is a Th cell coactivator, and its major ligand is MHC class II molecule. The binding of the second-class MHC molecule present on the surface of the antigen-presenting cell with CD4 is the second signal of Th cell activation.

The cells including CD4 on their surface are involved in the regulation of the immune response, either supporting it or inhibiting it. Depending on these 2 functions, they are respectively called helper and regulatory (suppressor). CD4+ cells are divided into 4 main groups: Th1, Th2, Th17, and Treg. Besides them, the following are distinguished: Tfh (follicular helper), Th9, and Th22 cells [9]. However, it is uncertain whether these CD4+ cell types constitute separate Th cell lineages or they are variants of major type cells, but they certainly display distinctive features [16].

\section{Th1 cells}

Th1 cells are developed from Th0 in the IL-12 and interferon- $\gamma(\mathrm{IFN}-\gamma)$ environment with the participation of the transcription factors T-bet (T box expressed in T cells) and STAT1 and STAT4 (signal traducer and activator of transcription) [14, 17-19].

The most powerful Th1-polarizing cytokine is IL-12, which is produced by myeloid dendritic cells (DCs) [19]. IL-12 not only induces the differentiation of Th1 lymphocytes, but also increases their activity and proliferation, and stimulates the secretion of IFN- $\gamma$ and tumour necrosis factor- $\alpha$ (TNF- $\alpha$ ). IL-12 is also important because it plays a negative role in the development of autoimmune diseases, and the monoclonal antibody against the p40 subunit of IL-12 is used in the treatment of psoriasis [14].

Th1 cells also play a decisive role in promoting a cell-type response. This is mainly done by IL-2, lymphotoxin $\alpha$ (LT- $\alpha)$, and IFN- $\gamma$, which are produced by Th1 $[15,20]$. The activated CD8+ cytotoxic cells, stimulated by these cytokines, are primarily responsible for the destruction of cells, including thyrocytes in HT.

\section{Th2 cells}

In order to obtain the second group of Th2 cells, both IL-2 and IL-4 must be present. The major product of Th2 lymphocytes, IL-4, is also a critical inducer of Th2 cell differentiation $[14,16]$. Th2 lymphocytes produce IL-4, IL-5, IL-10, and IL-13, which are factors in the B-lymphocyte growth and differentiation. Thus, their primary function is to promote a humoral immune response. Th2 cells play a major role in immune defence against extracellular parasites and development of an allergic reaction [20]. Interestingly, interleukins secreted by Th2 cells inhibit the production of inflammatory cytokines, such as IFN- $\gamma$. It was found in animal models that IL-4 had a protective effect on the development of the autoimmune diseases, which will be described later [7].

As in the Th1 lineage, as well as cytokines, transcription factors play an important role in the differentiation of Th2 lymphocytes. STAT6 (signal transducer and activator of transcription 6) and GATA-3 are the main transcription factors for the Th2 subtype [21,22]. STAT6 
is a downstream effector of IL-4, while GATA-3 is associated with the promotion of the secretion of Th2 cell cytokines such as IL-4, IL-5, and IL-13, thereby promoting a humoral response [23].

\section{Th17 cells}

Like Th1 and Th2 lymphocytes, Th17 cells develop from naive CD4 cells ( 1 out of 4 cell groups: Th1, Th2, Th17, and iTregs) $[14,16]$.

Th 17 cells were described in 2003 by Aggarwal [24]. The discovery of this lymphocyte subtype was initiated and named after the identification of IL-17A in 1993 (by Rouvier et al.) [25, 26].

They have been described as producing IL-17A, IL-17F [27], and IL-22 [28], which distinguishes them from Th1 and Th2 cells, which do not produce these cytokines [29]. Th17 also produces IL-21; however, it might be secreted by other Th cells [16]. Differentiation of naive T cells into Th17 cells is mainly induced in the environment of transforming growth factor-beta (TGF$\beta$ ) and IL-6, but other cytokines also contribute, such as IL-1 $\beta$, IL-21, IL-23, and TNF- $\alpha$ [30-32].

In addition, transcription factors contribute to the formation (differentiation) of Th17 from naive $\mathrm{T}$ cells. The most important of them are retinoic acid receptor-related orphan receptor $(\mathrm{ROR} \gamma \mathrm{t})$ and STAT3 and equally important STAT5, interferon regulatory factor-4 (IRF-4), and basic leucine zipper transcription factor ATF-like (BATF), Runt-related transcription factor-1 (Runx-1), and the aryl hydrocarbon receptor (Ahr) $[29,33]$. ROR $\gamma$ t remains the characteristic transcription factor allowing the identification of Th17 cells.

Dealing with Th17 cells, the existing division into non-pathogenic and pathogenic Th17 cells should be noted. The non-pathogenic Th17 cells naturally occur in the gut, and they are responsible for maintaining the homeostatic microbiota and fighting against pathogenic microbes [34]. They have no autoreactive features. Unlike non-pathogenic ones, the pathogenic Th17 cells arise in the presence of IL-23 and are mainly responsible for the development of autoimmune reactions to self-antigen [34, 35].

\section{Tfh cells}

As effector cells, the Tfh cells can enter tissues and mediate inflammation. Additionally, this population develops into memory cells residing in the lymph nodes and the spleen. These effector/memory cells are distinct states of each type: Th1, Th2, Th17, and Tregs. Their main function is to help B lymphocytes to produce antibodies, especially in the germinal centres, so they are called follicular helper cells Tfh [16]. These cells mediate both helping B lymphocytes and switching Ig classes (help and class switching).
Tfh cell differentiation takes place in several steps and requires the presence of IL-6, IL-12, IL-21, and activin A signalling [36]. Their main transcription factor is B cell lymphoma 6 (Bcl6), which limits Th1 differentiation and supports Tfh cell differentiation [37]. Bcl-6 controls expression of most of genes related to Tfh cell differentiation, but it is not sufficient for the development of these cells. Equally important is the expression of transcription factor Maf, also known as proto-oncogene c-Maf (c-Maf), which induces the production of IL-21 necessary for the formation of Tfh cells [36].

It is uncertain whether Tfh is an independent and individual CD4 lineage or an effector state of each of the 3 CD 4 cell lineages. Tfh cells produce both IL-4 and IFN- $\gamma$ as do Th1 and Th2, but we do not know if Tfh cells acquired the ability to produce IL- 4 and IFN- $\gamma$, or whether Th1 and Th2 acquire the features of Tfh cells. Pearce's studies showed that Tfh cells can arise from Th2 cells, which acquire the Tfh phenotype after antigen activation [16]. Thus, it is unknown whether this is a separate CD4+ cell lineage. However, studies confirm that dysregulation of Tfh cell differentiation and function may be associated with the development of autoimmunity [37].

\section{Th9 cells}

Relatively recently, Veldhoen et al. (2008) described a subpopulation of CD4 + cells that produce a lot of IL-9, and less IL-10 and IL-23 [38]. Primarily the product of these cells, IL-9 was discovered in 1989 by Van Snick et al. [39]. However, it was uncertain which of the known immune system cells produce the greatest amount of this cytokine, especially if it is secreted by several types of cells [40]. Initially, it was considered a product of Th2 cells. Just 13 years ago it was disclosed that the Th lymphocytes producing IL-9 in such large numbers do not have the ability to secrete cytokines typical of other known Th cell lines: IL-4, IL-5, and IL-13 of Th2; IFN- $\gamma$ for Th1; and IL-17 $\alpha$ for Th17. They also lack the specific transcription factors T-bet for Th1, GATA-3 for Th2, ROR- $\gamma$ t for Th17, and FoxP3 for Treg. Thus, they constitute a different T-lymphocytes subpopulation that was named Th9 lymphocytes [41]. These cells develop from naive CD4+ T cells in the presence of IL- 4 and TGF- $\beta$, which inhibit Th 2 cells [40].

The transcription key factor for Th9 lineage development is PU.1, which is one of the ETS family transcription factors (E-26), as well as IRF-4. The latter is induced by STAT6, which is activated by IL-4. Thus, the presence of STAT6, PU.1, and IRF-4 is characteristic of CD4+ Th9 cells. Th9 cells are involved in immunity to parasites, cancer (IL-9 is crucial in inhibiting tumour growth), and allergic diseases (mainly asthma) [14,41]. 
Th9-secreted IL-9 has a pleiotropic effect, but one of its functions is to stimulate Th17 proliferation and increase inflammation. In this way Th9 cells may be involved in the development of autoimmune diseases.

\section{Th22 cells}

A year after the description of Th9 cells, a new subset of IL-22-producing CD4+ cells was discovered (Trifari, 2009) [42]. Like the previous Th cell subtypes, Th22 cells also develop from naive CD4+ T cells, and their differentiation occurs in the presence of Il-6 and TNF- $\alpha$ [43]. A lot of earlier data show that IL-22 (described in 2000) is produced by activated T cells including Th22 cells, Th17 cells, Th1 cells, and the other lymphoid cells. Initially it was thought that IL-22 was a Th1-associated cytokine. After the discovery of Th17 cells, these lymphocytes were considered crucial in the production of IL-22. In subsequent studies it turned out that there was a separate lineage of Th cells, which produce IL-22 without IFN- $\gamma$, IL-4, and IL-17 $\alpha$, and its differentiation depends on the transcription factor aryl hydrocarbon receptor (AHR). AHR is a key feature of Th22 cells. Engagement of AHR leads to the increase of IL-22 production and the decrease of IL-17 expression [43]. IL-22 expression requires AHR. Interestingly, the receptors for IL-22 (IL-22R) are expressed in skin, small intestine, liver, and kidney but not in the immune system [9].

The main function of IL-22 is a regulation of various innate mechanisms to eliminate invasive pathogens attacking epithelial cells. IL-22 has been linked to skin homeostasis and inflammation [14, 43].

Th22 cells, through secreted IL-22, also exert a negative effect, i.e. they are closely associated with various diseases, including autoimmune diseases (psoriasis, rheumatoid arthritis, inflammatory bowel diseases, SLE, systemic sclerosis, and others) [16, 44, 45].

\section{Regulatory T-cell subtypes}

Regulatory lymphocytes (Tregs), described in the 1970s, are characterized by inhibition of the immune response. Tregs maintain homeostasis and prevent autoimmune responses by targeting $\mathrm{T}$ cells, dendritic cells, macrophages, mast cells, and B cells [46]. Their action is direct, from cell to cell, or through secreted cytokines such as TGF- $\beta$ and IL-10. What makes Tregs a distinctive separate group of CD4 + cells is high constitutive expression of the surface marker CD25, i.e. IL-2 receptor $\alpha$ chain, cytotoxic T-lymphocytes associated protein 4 (CTLA-4), and glucocorticoid-induced tumour necrosis factor-related protein (GITR), as well as the expression of the specific transcription factor forkhead box protein 3 (FoxP3) [47]. The FoxP3 mutation is responsible for the development of IPEX syndrome (immunodeficiency, polyendocrinopathy and enteropathy, X-linked syndrome).

Likewise, the CD4+ helper cell type, Treg lymphocytes also comprise a heterogeneous group.

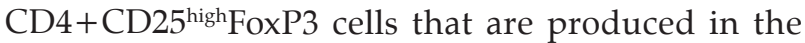
thymus are called natural (nTreg) or thymus-derived Treg cells $[11,14]$. The nTregs, completely differentiated in the thymus, can recognize self-antigens and inhibit the activation, proliferation, and synthesis of cytokines by effector lymphocytes [11].

The second group of Tregs lymphocytes are the cells that after contact with the antigen develop and differentiate peripherally, beyond the thymus, so they are called inducible Tregs (iTregs) or peripheral differentiated (pTregs) $[8,11]$. The iTreg cells differ from $n$ Tregs in the mechanism of immunosuppressive action, which is mediated by the secretion of TGF- $\beta$ and IL-10 cytokines, but not directly from cell to cell [11]. The cytokines IL-10 and TGF- $\beta$ secreted by iTregs can affect the cytokine production of CD4+ T cells. An increasing amount of evidence indicates that the iTreg subtype is not a homogeneous group, but is divided into 2 subgroups, named $\operatorname{Tr} 1$ and Th3, depending on the secreted cytokines. Tr1 cells are thought to secrete mainly IL-10, and Th3 cells secrete predominately TGF- $\beta$ [8].

In recent years, new groups of Treg lymphocytes have been described: the first of the CD69+ phenotype with different expression of CD25, IL-10, and TGF- $\beta$, as well as another of the CD4+NKG2D+ phenotype (NKG2D is an activating receptor expressed by all NK cells and subsets of T lymphocytes). The latter play a significant immunosuppressive role, which is apparently mediated by TGF- $\beta$ and IL-10 [48].

The main function of Treg is to inhibit autoreactive $\mathrm{T}$ lymphocytes, so it is obvious that this cell lineage defect will have an impact on the development of autoimmune diseases.

\section{Relationships between CD4+ cell lineages}

The differentiation and function of a particular CD4+ cell lineage is primarily dependent on the presence of specific transcription factors [21-23]. Overexpression of T-bet during Th2 differentiation causes such cells to acquire competence to produce IFN- $\gamma$ while, at the same time, suppressing their capacity to produce IL-4 [16]. It has also been shown that IRF-4 (IFN regulatory factor- 4 , which is important in Th2 differentiation, also plays a role in the differentiation of Tregs controlling Th2. Similarly, it seems that STAT3 expression in Tregs is necessary for the ability to suppress the pathological immune response by Th17 cells, which require the presence of STAT3 for differentiation $[14,16]$. Therefore, iTreg cells do not need to be considered as one of the 


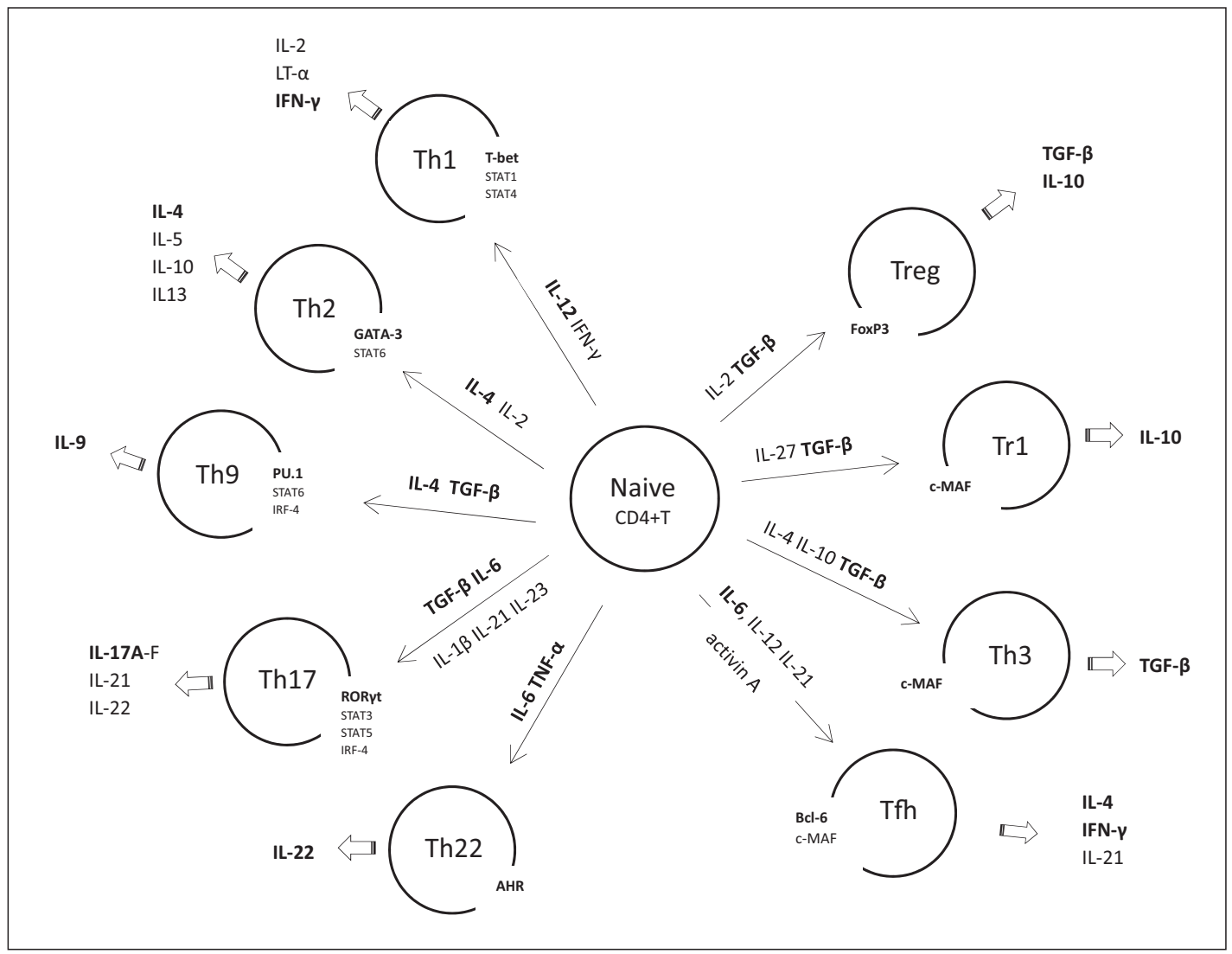

Figure 1. Differentiation and features of Th cell subtypes. IL - interleukin; LT- $\alpha$ - lymphotoxin; IFN- $\gamma$ - interferon gamma; TGF- $\beta$ - transforming growth factor beta; TNF- $\alpha$ - tumour necrosis factor alpha

CD4 cell distinct fates (like Th1, Th2, and Th17), but as a possible parallel line capable of separate regulatory targets [16].

In addition to transcription factors, important in the distinct differentiation of CD4 cells, the cytokine environment plays a major role in cell differentiation. The dependence of the effects of cytokines in individual CD4+ cell lineages on the differentiation and function of the other cell lineages is the same as that of specific transcription factors from one group on another. Cytokines that promote the differentiation of one cell subtype have an inhibitory effect on the formation of another CD4+ cell subpopulation. For example, IL-4, which is necessary for Th2 lineage differentiation, is a factor that inhibits Th1 development. Consecutively, Th1 is formed in the presence of IL-12 and provided that IL-4 is neutralized [31]. IL-2 is a key repressor of Th17 differentiation [34].

The key role in the development of immune response, including autoimmunity, plays the lymphocyte plasticity phenomenon. On account of this, a specific subtype of CD4+ cells can differentiate into cells of a different subpopulation. All of this occurs under certain molecular conditions and in the presence of a specific set of cytokines. Thus, Th1 lymphocytes can transform into Th2 or Treg, Th2 into Th1, Th17, and Treg, Th17 into Th1, Th2, or Treg, and Treg into Th1, Th2, or Th17 [14,49].

\section{The importance of the Th and Treg lymphocyte subpopulations in AITD}

\section{Importance of Th1 and Th2 in AITD}

Cytokines secreted by Th1 lymphocytes, with a significant pro-inflammatory effect, have a clear influence on the development of autoimmune diseases, including AITD. Their main role has been described in the development of Hashimoto's thyroiditis; however, they also contribute in the development of Graves' Disease [50].

Hashimoto's disease is a classic example of a T-cell-mediated disease in which thyroid tissue is destroyed by an inflammatory infiltrate consisting of CD8+ and CD4+ T cells, CD19+ B cells, macrophages, and plasma cells [8]. Activated and recruited Th1 cells may be responsible for enhanced IFN- $\gamma$ and TNF- $\alpha$ production [10]. IFN- $\gamma$ increases the expression of MHC class I and II molecules, which significantly enhances the presentation of antigens to T lymphocytes, and thus it stimulates their cytotoxicity. IFN- $\gamma$ is also the strongest activator of macrophages, which results in the 


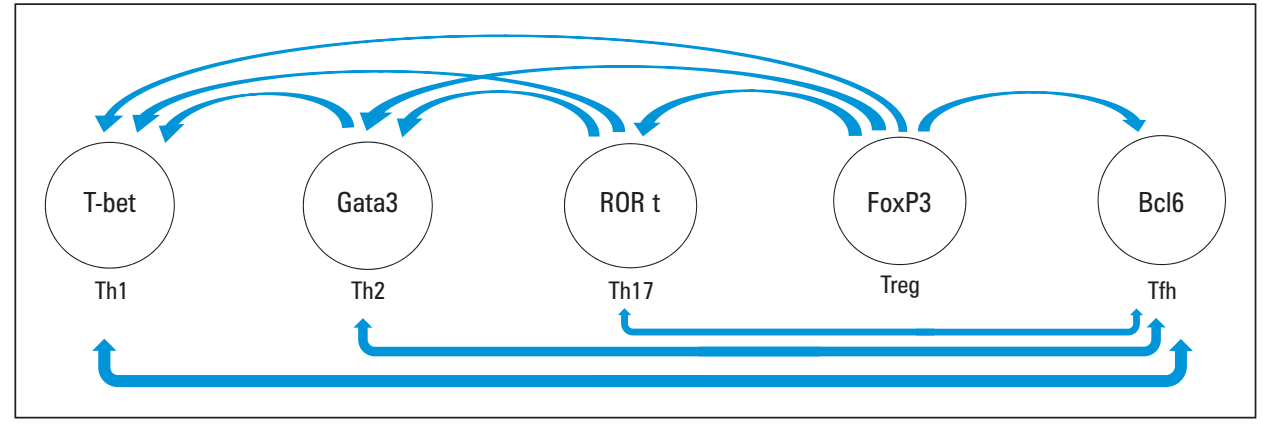

Figure 2. Plasticity of Th cells

intensification of their cytotoxicity and the destruction of thyrocytes $[8,14]$.

Similarly to IFN- $\gamma$, TNF- $\alpha$ is one of the major cytokines in inflammation. TNF- $\alpha$ increases the proliferation of $\mathrm{T}$ and $\mathrm{B}$ lymphocytes, induces the secretion of many cytokines (including IFN- $\gamma$ ), activates macrophages, and has a strong chemotactic effect [14]. In the presence of increased amounts of IFN- $\gamma$ and TNF- $\alpha$, the thyroid cells secrete the chemokine CXCL10, which intensifies the inflammatory infiltrate and enhances the autoimmune process. High levels of circulating CXCL10 have been shown in patients with AITD, mainly in HT, and circulating CXCL10 is associated with the active phase of GD and GO [10].

It was discovered that the proportions of peripheral Th1 cells in CD4+ cells in patients with severe HT were significantly higher than those with mild HT [7]. Moreover, it was found that IFN- $\gamma$ polymorphism, which is related to higher IFN- $\gamma$, is more frequent in patients with severe HT than in those with mild HT [51].

Qiu Qin et al. proved that IFN- $\gamma$ mRNA levels are higher in the peripheral blood of patients with HT compared to healthy controls. Likewise, the mRNA levels of IFN- $\gamma$ were higher in patients with GD than in normal controls, but the difference was not significant [52].

In addition to the cytotoxic action of Tlymphocytes, the second mechanism of thyrocyte destruction in HT is through death receptors, which allow transmission of the apoptotic signal into the cell. One of the death receptors is the FAS receptor (CD95), the ligand of which is FasL. The combination of Fas and FasL leads to programmed cell death (apoptosis). There is evidence that thyrocytes from HT patients have the expression of both FAS and FAS ligand. The third mechanism of thyrocyte destruction is through anti-Tg and anti-TPO antibodies. The thyroid cells damaged by antibodies release cytokines such as IL-6, IL- $1 \beta$, and IL- 8 , which further stimulate the cytotoxic effects of lymphocytes. It is currently uncertain whether the presence of the antibodies is the cause or the effect in the destruction of thyrocytes [8].
Certainly, anti-thyrotropin receptor antibodies (TRAbs) are the cause of the development of Graves' disease. Therefore, this disease is classically described as a $B$ cell-mediated disease. The interaction between $\mathrm{CD} 4+\mathrm{T}$ cells and auto-reactive B cells is required for the production of autoantibodies against the TSHR. Th2-mediated immune response is involved in this process [53].

On the other hand, Th2 cells produce cytokines including IL-4, IL-5, and IL-10, which may play a protective role in GD. It was shown above that in animal models IL-4 inhibits the development of autoimmune diseases [7]. In Hashimoto's disease, Nanba et al. reported that $-590 \mathrm{CC}$ genotype in $-590 \mathrm{C} / \mathrm{T}$ polymorphism of the IL-4 gene, which is related to lower IL-4 production, was more frequent among severe HT patients compared to mild HT patients [54]. Similarly, in Graves' disease, IL-4 and IL-10 induce T-cell anergy, inhibit IFN- $\gamma$ secretion and CD8 + T cell cytotoxicity, and induce a phenotypic switch from Th1 to Th2 [53].

The imbalance of Th1 and Th2 is therefore very important in the development of both HT and GD. The imbalance into Th1 increases the destruction of thyrocytes, gland atrophy, and the development of hypothyroidism, while the imbalance into Th2 results in the appearance of stimulating TRAb and causes hyperthyroidism and enlargement of the gland.

\section{The importance of Th17 in AITD}

Over the past 15 years, Th17 cells have emerged as crucial in the development of autoimmune diseases [32, 55]. The IL-17 secreted by them stimulates the following (this family includes 6 interleukins described with letters from A to F, and IL-17E - also called IL-25): epithelial cells, fibroblasts, and macrophages for producing inflammatory mediators such as chemokines, cytokines TNF- $\alpha$, IL-1 $\beta$, IL-6, IL-8, GM-CSF, and MCP-1 (monocyte chemotactic protein) [35]. IL-17A and IL-17F are also key cytokines for the recruitment, activation, and migration of neutrophils [56]. All this makes Th17 cells one of the most productive effector cells among $\mathrm{T}$ lym- 


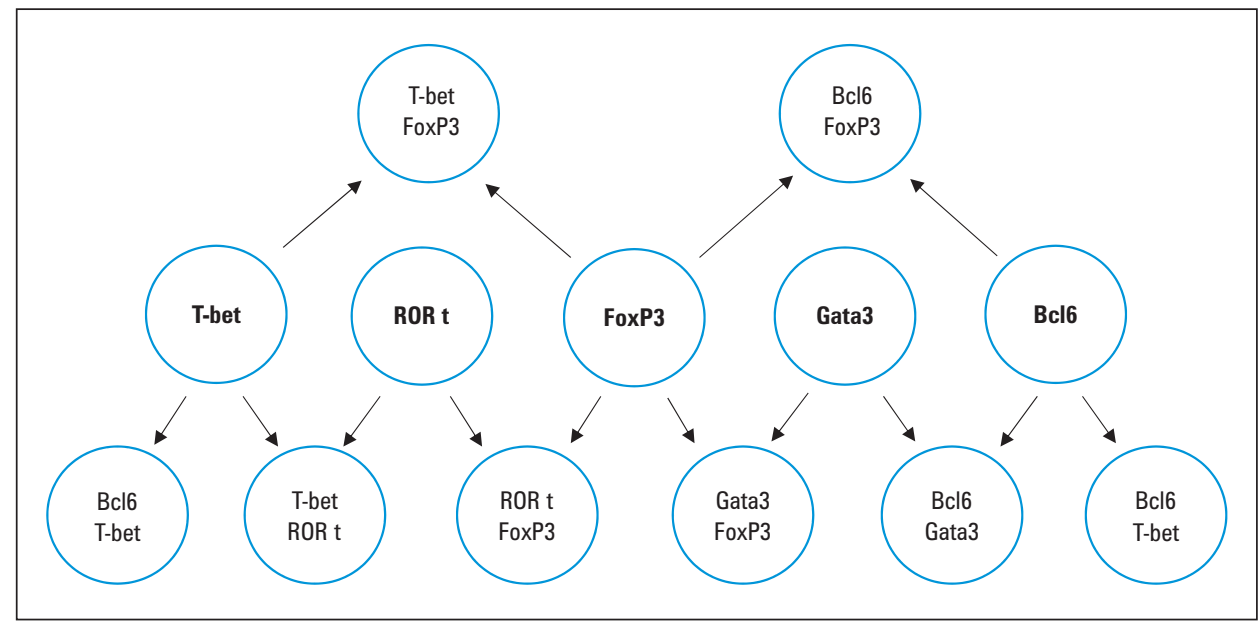

Figure 3. Flexibility of Th cells

phocytes. Numerous studies have found an increased percentage of Th17 cells and the cytokines of patients suffering from multiple autoimmune diseases, such as type 1 diabetes [17], inflammatory bowel disease [57], systemic lupus erythematosus, rheumatoid arthritis, multiple sclerosis, and psoriasis [58].

In the last decade, an increased share of Th17 cells and the cytokines they secrete in the course of autoimmune thyroid diseases has also been described many times $[7,59,60]$. Researchers found enhanced levels of T cells synthesizing IL-17 and IL-22 in the peripheral blood from AITD patients, mainly in those with HT. Interestingly, clearly a stronger expression of IL-17 and IL-22 was detected in the thyroid glands of HT patients in comparison with Graves' disease patients or controls [60]. These studies indicate increased involvement of Th17 cells in the pathogenesis of AITD, especially HT. Similar conclusions can be drawn from the already cited research by Qui Qin et al., in which, besides Th1 cell cytokines, the levels of IL-17A and IL-23 in thyroid tissues were also studied. Tissue expression of IL-17A and IL-23 was significantly higher in the HT group compared to the control group, but it was not significantly increased in the GD group [52].

Scholars from Latvia also described the lack of a significant increase in the tissue expression of IL-23 in the group of patients with GD compared to the control group. Tatjana Zake et al. investigated the expression of IL-17 as well as IL-23 and IL- $1 \beta$ in the thyroid tissue of patients with HT and GD and patients with goitre as a control group. They found that the expression level of IL-17 in thyrocytes was significantly higher in both HT and GO patients than in colloid goitre patients, but IL-23 and IL-1 $\beta$ expression was increased only in the HT group, not in the GD group [61].

Cui Li et al. in 2016 also estimated the presence of Th17 cells in AITD, dividing patients into an HT (Hashi- moto's thyroiditis) group, a GD (Graves 'disease) group, and a GO (Graves' ophthalmopathy) group. They found that compared with those in the control group, the percentage of CD4+IL-17+T cells and mRNA expression of its transcription factor ROR $\gamma \mathrm{t}$ were higher in peripheral blood mononuclear cells of AITDs, also particularly in the HT subgroup. It is worth emphasising that in the GO group in patients with CAS above 4.5 the percentage of Th17 was significantly increased compared to those with CAS below 4.5, which indicates the degree of Th17 cell involvement in the inflammatory process depending on its activity [62].

A study by Vitales-Noyola $\mathrm{M}$ et al., which we mention below, also confirmed the dependence of Th17 cells on disease activity. They found increased levels of pathogenic Th17 cells in the peripheral blood and thyroid glands of AITD patients, and the level of pathogenic Th17 cells was dependent on the activity of AITD [63].

One of the highly pro-inflammatory cytokines, secreted mainly by macrophages and monocytes after IL-17A simulation, and secreted also by Th17 cells alone, is IL- $1 \beta$. This cytokine simultaneously promotes (in addition to IL-6, IL-21, and IL-23) the formation of Th17 lymphocytes [35]. Japanese researchers conducted a study on the polymorphism of the gene encoding IL- $1 \beta$. They found that the $-31 \mathrm{C} / \mathrm{T}$ polymorphism of the IL- $1 \beta$ gene, which is related to the high producibility of IL- $1 \beta$, was significantly more frequent in patients with intractable GD than in those with GD in remission. Additionally, they showed that the proportion of IL-17-producing Th17 cells was higher in autoimmune thyroid disease patients with the $-31 \mathrm{C} / \mathrm{T}$ polymorphism [64].

Li Zheng et al. proved that the expression of ROR $\gamma \mathrm{t}$, IL-17 mRNA, and IL-17 protein levels were markedly higher in GD patients group as compared with the 
control group. Interestingly, high levels of IL-17 were found in the group of GD patients in hyperthyroidism and in the euthyroid GD group. The same authors also investigated the expression of ROR $\gamma \mathrm{t}$ and the levels of IL-17 mRNA and the protein after the administration of IL-23. The expression of ROR $\gamma \mathrm{t}$, and levels of IL-17 mRNA and protein in the GD group with IL-23 dramatically increased as compared with that in the GD group without IL-23 and in the control group without IL-23 [65].

This study confirms the enhancement of proliferation and function of Th17 cells by IL-23, and that persistent exposure to IL-23 triggers the conversion of classical Th17 cells into pathogenic Th17 cells [35].

The role of Th17 lymphocytes in the development of AITD is beyond doubt. An increasing amount of evidence points to the participation of this group of cells in the development of AITD, especially HT; however, the data concerning the participation in the pathogenesis of GD are not equivocal. It seems that the role of Th17 in the development of GD is not as crucial as in the development of HT, where pathogenic Th17 cells stimulated by IL-23 dominate among CD4+ cells.

\section{The importance of Th9 and Th22 in AITD}

In recent years, the involvement of IL-9 and Th9 cells in the pathogenesis of rheumatoid arthritis [66], systemic lupus erythematosus, SLE [67], multiple sclerosis [68] and systemic sclerosis [69] has been described. In patients with these diseases, increased serum IL-9 was found [41].

Unfortunately, there are few data on the involvement of Th9 cells in the pathogenesis of AITD. An interesting study was conducted by Zivancevic-Simonovic et al. in 2015. They found that papillary thyroid cancer (PTC) patients with HT produced significantly higher concentrations of IL-9 than PTC patients without HT, which indicates the role of Th9 cells in the pathogenesis of AITD [70]. There are also experimental studies of autoimmune diseases with anti-IL-9 treatments [14,71].

We know more about the involvement of Th22 cells in the development of AITD. As mentioned above, Th22 cells and IL-22 have been considered to participate in the pathogenesis of numerous autoimmune diseases. There are also some data on the involvement of these cells in the development of AITD. Peng D et al. confirmed a higher frequency of IL-22 $+\mathrm{CD}+\mathrm{T}$ cells and higher concentrations of serum IL-22 in Graves's disease (GD) patients. Moreover, they found a positive correlation between the percentages of Th22 in peripheral blood with the concentrations of serum TRAb in GD patients [72].

Similar conclusions, but extended to patients with HT, were obtained by Vitales-Noyola et al. in 2017. They found increased levels of Th22 cells in the peripheral blood of autoimmune thyroid disorder patients and increased presence of Th22 cells in the thyroid glands of Hashimoto's thyroiditis patients. Additionally, they found significant correlations between the levels of Th22 cells and disease activity, disease duration, and the presence of ophthalmopathy [63].

The involvement of Th22 cells in the pathogenesis of AITD was also investigated by Bai et al. The authors showed the level of circulating Th22 cells correlated with the level of serum IL-22 in patients with HT and was significantly higher than in GD patients and healthy control subjects [73].

Despite the undoubted role of Th22 lymphocytes in the pathogenesis of AITD, it seems that their role is one of many and is not crucial for the development of AITD. Further research on this group of cells is certainly needed.

\section{The importance of Treg in AITD}

The number of studies on the role and importance of Treg lymphocytes in the development of AITD has clearly increased in the last decade. As is shown below, this is caused by the growing interest in Th17 cells, which are crucial in the development of AITD, and whose proliferation and function is tightly controlled by Treg lymphocytes.

It was assumed that in autoimmune diseases a reduced share of Tregs lymphocytes would be observed, which have an immune response suppression function. However, the research showed a slightly different, less expected result.

In murine models, depletion of CD4+CD25+ Tregs enhances the incidence and severity of HT or GD. McLachlan et al. proved that this depletion in genetically manipulated mice could induce a shift from GD to HT [5]. In addition, prior transfer of CD4+CD25+ Treg cells into mice could suppress Tg-induced experimental autoimmune thyroiditis (EAT) [35].

Similar results have been obtained in studies on humans. In the study by Cui Li et al., besides examining Th17 cells, the authors also determined the number of Treg cells in patients with AITD. According to their results, the percentage of $\mathrm{CD} 4+$ Foxp $3+\mathrm{T}$ cells and the mRNA expression of its transcription factor Foxp3 were significantly decreased in peripheral blood mononuclear cells (PBMCs) of AITD patients. However, a clear reduction of Treg cells was observed only in the GD group, while in the HT and GO groups the difference compared to the control group was less significant [62].

Diminished levels and defective suppressive function of $\operatorname{Tr} 1$, one of the subtypes of T regulatory lymphocytes in patients with autoimmune thyroiditis, was shown by Marlen Vitales-Noyola et al. in 2018 [74]. 
Conversely, an increased presence of Treg cells in patients with AITD was already observed in studies 2 decades ago. Monica Marazuela et al. explored the proportion of different T-cell subsets with regulatory phenotype in the peripheral blood of patients with AITD and healthy controls. They confirmed a significantly higher proportion of CD4+GITR+, CD4+Foxp3+,CD4+CD69+, and CD4+ cells synthesizing IL-10 or TGF- $\beta$ (respectively, Tr1 cells and Th3 cells) in patients with AITD. The scholars also found an increased percentage of Treg phenotype cells in thyroid tissue from patients with autoimmune thyroiditis. Interestingly, they assessed the suppressive function of CD4+CD25+Treg cells using different cell proliferation assays, and they found a significantly diminished regulatory activity of Treg cells from AITD patients, compared with controls [75]. According to these data, the clearly increased presence of Treg lymphocytes in the thyroid of AITD patients is unable to inhibit the self-destruction process, which indicates an impairment of Treg function in the pathogenesis of AITD.

Continuing this direction of research in 2015 Rodríguez-Muñoz et al. reached similar conclusions. This time, the presence of a new group of Treg lymphocytes with the phenotype CD4+CD69+ and CD4+NKG2D+ secreting IL-10 and TGF- $\beta$ in patients with AITD was investigated. They found a significantly increased percentage of these Tregs cells in peripheral blood and thyroid tissue from GD, especially with GO patients compared to controls. Importantly, positive correlation between GO activity and CD4+CD69+IL-10+ or CD4+CD69+IL-10+NKG2D+ cells was also found. Their findings suggest that increased levels of Treg cells in AITD patients are probably unable to down-modulate the autoimmune response [76].

Ziyi Chen et al. published last year a meta-analysis examining the percentage of peripheral blood Tregs among CD4+ T cells in patients with autoimmune thyroid disease. They found that the treatment status might affect the level of Tregs, so they conducted this meta-analysis dividing AITD patients into untreated and treated groups. For example, among 10 studies of untreated AITDs, 7 reported significantly decreased proportions of circulating Tregs in AITDs compared to controls, 1 revealed non-significantly lower proportions of Tregs, and 2 studies revealed increased proportions of Tregs. The result of this study indicated that the proportions of circulating Tregs among CD4+ T cells of untreated AITDs were remarkably lower than in healthy controls, but no significant difference was found in treated AITDs [46].

These inconclusive data indicate that Tregs lymphocytes are potentially involved in the pathogenesis of AITD, but their function appears to be different in HT and GD and dependent on treatment status. The percentage of Tregs can change over the course of different stages of treatment. Certainly, the suppressive capacity of Tregs is impaired in both GD and HT [35].

What seems to be the most important is that the involvement of Tregs in the development of AITD should not be considered separately, but in relation to effector cells, mainly Th17 cells.

\section{Th17/Treg balance as a basis for AITD development}

The main role in the development of the autoimmune response is played by the Th17 and Th1 effector cells and, on the other hand, the inhibitory Tregs lymphocytes. Based on current knowledge, the mutual balance between Th17 cells and Tregs is the basis for the proper functioning of the immune system, and the imbalance is the crucial cause of the development of autoimmune diseases, including AITD. The deviated balance between Treg and Th17 with an orientation to Th17 in an animal model of autoimmune disease and in most human autoimmune diseases was confirmed [77].

Several studies have shown an increased ratio of Th17/Treg in patients with AITD. Li et al., concluding a Th/Treg balance study in different AITD subtypes, found that increased Th17 lymphocytes may play a more important role in the pathogenesis of HT and GO, while decreased Treg may be greatly involved in GD [62].

Qin et al. studied the involvement of Th17 and Treg cells in new-onset Graves' disease. They detected a significantly decreased proportion of circulating CD4+Foxp3+Treg cells and reduced Foxp3 mRNA expression in patients with new-onset GD. The reduction of circulating Treg cells may be due to the increased apoptosis of these cells found in previous studies by Nakano et al. and Mao et al. The authors also confirmed that with the lower share of Treg cells, there is an increased percentage of Th17 cells, and enhanced IL-17 and ROR $\gamma \mathrm{t}$ mRNA expression level in patients with new-onset GD [78].

Moreover, a positive correlation was found between the proportion of circulating Th17 cells and serum TRAb activity. Comparing the occurrence of Th17 and Treg cells, researchers found that the ratio of circulating Th17/Tregs was significantly higher in GD patients than in healthy controls [78].

Whether it is the increased involvement of Th17 cells or the decreased participation of Treg cells that causes the development of particular AITD subtypes, the imbalance of Th17/Tregs seems to be most important in the development of AITD pathogenesis. 


\section{Mutual regulation between Th17 and Treg}

Based on the knowledge that the imbalance between cytotoxic Th17 cells and regulatory T cells is the foundation for the development of AITD, the factors regulating the correlation of Th17 with Treg cells can be expected to play a key role.

The main modulator of Th17/Treg balance is transforming growth factor- $\beta$. TGF- $\beta$ in the presence of IL- 6 , and IL- $1 \beta$ plays an important role in the differentiation of IL-17 producing T cells from naive CD4+ T cells [79]. Moreover, TGF- $\beta$ not only leads to the development of the Th17 cell lineage by inducing ROR $\gamma \mathrm{t}$, but also inhibits the differentiation of Th1 and Th2 cells [77, 79]. On the other hand, TGF- $\beta$ in the IL- 2 environment upregulates Foxp3 expression and induces Treg cell differentiation [77]. The Tregs secrete TGF- $\beta$ in addition to IL-10; therefore, TGF- $\beta$ promotes the formation of further Tregs, but TGF- $\beta$ participates in creating of the Th17 lineage only in the environment of proinflammatory cytokines such as IL-6, IL-1 $\beta$, IL-23, etc. [17, 77]. The effect of TGF- $\beta$ on the possible expression of both $\mathrm{ROR} \gamma \mathrm{t}$ and Foxp3 indicates the presence of a common precursor cell ROR $\gamma \mathrm{t}+$ Foxp3+, which in the environment of lineage-specific polarizing factors differentiates into Th17 or Tregs $[16,80]$.

In addition to TGF- $\beta$, also retinoic acid (vitamin A metabolite) and lipid inflammatory mediators such as leukotriene B4 (LTB4) and prostaglandin E2 (PGE2) as well as macro bubbles (MV) and SLAMF1 (signaling lymphocyte activation molecule family molecule family 1) have a modulating effect on the Th17/Treg balance $[35,77]$. (signalling lymphocytic activation molecule family molecule family 1 ) have a modulating effect on the Th17/Treg balance $[35,77]$. Although this issue is not the scope of our work, it shows the complexity of the process of maintain Th17/Treg balance.

\section{CD4+ lymphocytes - plasticity and phenotype variability}

All CD4+ cells have the capacity to change plasticity and phenotype (flexibility). Interestingly, it seems that Th17 and Treg cells have the greatest possibility of changing the phenotype. In the presence of IL-12, Th17 cell, that secreting IL-17 and IFN- $\gamma$ (Th17/Th1 cell), could be transformed into a cell secreting only IFN-g, called non-classic Th1 ${ }_{\alpha}$ can be formed into a cell secreting only IFN- $\gamma$, called non-classic Th1. It is also known that there are Th17 cells producing IL-4 (Th17/Th2 cell), besides IL-17 [81].

The common precursor ROR $\gamma \mathrm{t}+$ Foxp3 + cell, playing the opposing role of Th17 and Treg cells, is an example of the heterogeneity and potential variability of CD4+ cells [16]. It has been shown that CD4+Foxp3+ cells can transform into IL-17 producing lymphocytes in the environment of IL-2, IL-15, IL-21, and IL-23. An increased frequency of Foxp3+IL-17+ and Foxp3+IFN- $\gamma$ + lymphocytes was also found in patients with autoimmune diseases [11]. The possibility of converting immune suppression cells into pathogenic Th17 and vice versa is extremely interesting from a practical point of view and can be used in treatment.

\section{Conclusions}

Autoimmune thyroid disease is one of the most common autoimmune diseases. Activation of CD4+ cells is the primary cause of both thyrocyte damage and the production of TSHR-stimulating antibodies in autoimmune thyroid diseases. There is expanding knowledge on new CD4+ T cell subtypes involved in the pathomechanism of AITD. The essential factor was the shift from the Th1/Th2 balance paradigm to the fact that the Th17 and Treg imbalance is crucial for the development of AITD. In addition, the mutual relationship between CD4+ cells, their phenotypic variability, and the comprehension of factors influencing the balance, proliferation, and activation of CD4+ T cells is of great importance. Increasing knowledge of the role and importance of CD4+ cells in the pathogenesis of AITD allows not only a better understanding of the mechanisms of development of these diseases, but also helps to define therapeutic targets.

\section{Acknowledgments}

This work was supported by the Medical University of Silesia (grants no. KNW-1-186/N/8/K, KNW-1-133/N/9/K).

\section{References}

1. Locatelli F, Rondelli D, Burgio GR. Tolerance and hematopoietic stem cell transplantation 50 years after Burnet's theory. Exp Hematol. 2000; 28(5): 479-489, doi: 10.1016/s0301-472x(00)00153-3, indexed in Pubmed: 10812237.

2. Janeway CA, Janeway CA. Approaching the asymptote? Evolution and revolution in immunology. Cold Spring Harb Symp Quant Biol. 1989; 54 Pt 1(9): 1-13, doi: 10.1101/sqb.1989.054.01.003, indexed in Pubmed: 2700931.

3. Broggi A, Tan Y, Granucci F, et al. Microbe- and danger-induced inflammation. Mol Immunol. 2015; 63(2): 127-133, doi: 10.1016/j.molimm.2014.06.037, indexed in Pubmed: 25037632.

4. Matzinger P. Tolerance, danger, and the extended family. Annu Rev Immunol. 1994; 12: 991-1045, doi: 10.1146/annurev.iy.12.040194.005015, indexed in Pubmed: 8011301.

5. Pradeu T, Cooper EL. The danger theory: 20 years later. Front Immunol. 2012; 3: 287, doi: 10.3389/fimmu.2012.00287, indexed in Pubmed: 23060876.

6. Gonzalez-Diaz SN, Sanchez-Borges M, Rangel-Gonzalez DM, et al. Chronic urticaria and thyroid pathology. World Allergy Organ J. 2020; 13(3): 100101, doi: 10.1016/j.waojou.2020.100101, indexed in Pubmed: 32180891.

7. Nanba T, Watanabe M, Inoue N, et al. Increases of the Th1/Th2 cell ratio in severe Hashimoto's disease and in the proportion of Th17 cells in intractable Graves' disease. Thyroid. 2009; 19(5): 495-501, doi: 10.1089/thy.2008.0423, indexed in Pubmed: 19415997.

8. Kristensen B. Regulatory B and T cell responses in patients with autoimmune thyroid disease and healthy controls. Dan Med J. 2016; 63(2): B5177.

9. Li Q, Wang B, Mu K, et al. The pathogenesis of thyroid autoimmune diseases: New T lymphocytes - Cytokines circuits beyond the Th1-Th2 
paradigm. J Cell Physiol. 2019; 234(3): 2204-2216, doi: 10.1002/jcp.27180, indexed in Pubmed: 30246383

10. Antonelli A, Ferrari SM, Corrado A, et al. Autoimmune thyroid disorders. Autoimmun Rev. 2015; 14(2): 174-180, doi: 10.1016/j.autrev.2014.10.016, indexed in Pubmed: 25461470.

11. González-Amaro R, Marazuela M. T regulatory (Treg) and T helper 17 (Th17) lymphocytes in thyroid autoimmunity. Endocrine. 2016; 52(1): 30-38, doi: 10.1007/s12020-015-0759-7, indexed in Pubmed: 26475497.

12. Espinosa JR, Wheaton JD, Ciofani M. In Vitro Differentiation of CD4 T Cell Effector and Regulatory Subsets. Methods Mol Biol. 2020; 2111: 79-89, doi: 10.1007/978-1-0716-0266-9 7, indexed in Pubmed: 31933200.

13. Maier E, Duschl A, Horejs-Hoeck J. STĀT6-dependent and-independent mechanisms in Th2 polarization. Eur J Immunol. 2012; 42(11): 2827-2833, doi: 10.1002/eji.201242433, indexed in Pubmed: 23041833.

14. Gołąb J, Jakóbisiak M, Lasek W. Immunologia. VII, dodruk 2. Wydawnictwo Naukowe PWN, Warszawa 2017

15. Yin L, Zeng C, Yao J, et al. Emerging Roles for Noncoding RNAs in Autoimmune Thyroid Disease. Endocrinology. 2020; 161(8), doi: 10.1210/endocr/bqaa053, indexed in Pubmed: 32270194.

16. Zhu J, Yamane H, Paul WE. Differentiation of effector CD4 T cell populations $\left(^{*}\right)$. Annu Rev Immunol. 2010; 28: 445-489, doi: 10.1146/annurev-immunol-030409-101212, indexed in Pubmed: 20192806.

17. Piekarski R, Szewczyk L. Rola limfocytów Th17 w cukrzycy typu 1. Pediatric Endocrinol. 2011; 4(37)

18. Bedoya SK, Lam B, Lau K, et al. Th17 cells in immunity and autoimmunity. Clin Dev Immunol. 2013; 2013: 986789, doi: 10.1155/2013/986789, indexed in Pubmed: 24454481.

19. Romagnani S. Human Th17 cells. Arthritis Res Ther. 2008; 10(2): 206, doi: 10.1186/ar2392, indexed in Pubmed: 18466633.

20. Isailovic N, Daigo K, Mantovani A, et al. Interleukin-17 and innate immunity in infections and chronic inflammation. J Autoimmun. 2015; 60: 1-11, doi: 10.1016/j.jaut.2015.04.006, indexed in Pubmed: 25998834.

21. Murga-Zamalloa C, Wilcox RA. GATA-3 in T-cell lymphoproliferative disorders. IUBMB Life. 2020; 72(1): 170-177, doi: 10.1002/iub.2130, indexed in Pubmed: 31317631.

22. Tindemans I, Serafini N, Di Santo JP, et al. GATA-3 function in innate and adaptive immunity. Immunity. 2014; 41(2): 191-206, doi: 10.1016/j. immuni.2014.06.006, indexed in Pubmed: 25148023.

23. Maier E, Duschl A, Horejs-Hoeck J. STAT6-dependent and -independent mechanisms in Th2 polarization. Eur J Immunol. 2012; 42(11): 2827-2833, doi: 10.1002/eji.201242433, indexed in Pubmed: 23041833.

24. Aggarwal S, Ghilardi N, Xie MH, et al. Interleukin-23 promotes a distinct CD4 T cell activation state characterized by the production of interleukin-17. J Biol Chem. 2003; 278(3): 1910-1914, doi: 10.1074/jbc.M207577200, indexed in Pubmed: 12417590.

25. Rouvier E, Luciani MF, Mattéi MG, et al. CTLA-8, cloned from an activated $\mathrm{T}$ cell, bearing AU-rich messenger RNA instability sequences, and homologous to a herpesvirus saimiri gene. J Immunol. 1993; 150(12): 5445-5456, indexed in Pubmed: 8390535.

26. Sergejeva S, Lindén A. Impact of IL-17 on cells of the monocyte lineage in health and disease. Endocr Metab Immune Disord Drug Targets. 2009; 9(2): 178-186, doi: 10.2174/187153009788452444, indexed in Pubmed: 19519466

27. Harrington LE, Hatton RD, Mangan PR, et al. Interleukin 17-producing CD4+ effector $\mathrm{T}$ cells develop via a lineage distinct from the $\mathrm{T}$ helper type 1 and 2 lineages. Nat Immunol. 2005; 6(11): 1123-1132, doi: 10.1038/ni1254, indexed in Pubmed: 16200070

28. Dumoutier L, Van Roost E, Ameye G, et al. IL-TIF/IL-22: genomic organization and mapping of the human and mouse genes. Genes Immun. 2000; 1(8): 488-494, doi: 10.1038/sj.gene.6363716, indexed in Pubmed: 11197690.

29. Beurel E, Lowell JA, Jope RS, et al. Th17 cells in depression. Brain Behav Immun. 2018; 69: 28-34, doi: 10.1016/j.bbi.2017.08.001, indexed in Pubmed: 28779999.

30. McGeachy MJ, Bak-Jensen KS, Chen Yi, et al. TGF-beta and IL-6 drive the production of IL-17 and IL-10 by T cells and restrain T(H)-17 cell-mediated pathology. Nat Immunol. 2007; 8(12): 1390-1397, doi: 10.1038/ni1539, indexed in Pubmed: 17994024

31. Burgler S, Ouaked N, Bassin C, et al. Differentiation and functional analysis of human T(H)17 cells. J Allergy Clin Immunol. 2009; 123(3): 588-95, 595.e1, doi: 10.1016/j.jaci.2008.12.017, indexed in Pubmed: 19178935.

32. Sutton CE, Lalor SJ, Sweeney CM, et al. Interleukin-1 and IL-23 induce innate IL-17 production from gammadelta T cells, amplifying Th17 responses and autoimmunity. Immunity. 2009; 31(2): 331-341, doi: 10.1016/j.immuni.2009.08.001, indexed in Pubmed: 19682929.

33. Ochs HD, Oukka M, Torgerson TR. TH17 cells and regulatory T cells in primary immunodeficiency diseases. J Allergy Clin Immunol. 2009; 123(5): 977-83; quiz 984, doi: 10.1016/j.jaci.2009.03.030, indexed in Pubmed: 19410687.
34. Yasuda K, Takeuchi Y, Hirota K, et al. The pathogenicity of Th17 cells in autoimmune diseases. Semin Immunopathol. 2019; 41(3): 283-297, doi: 10.1007/s00281-019-00733-8, indexed in Pubmed: 30891627.

35. Shao S, Yu X, Shen L. Autoimmune thyroid diseases and Th17/Treg lymphocytes. Life Sci. 2018; 192: 160-165, doi: 10.1016/j.lfs.2017.11.026, indexed in Pubmed: 29158050.

36. Jogdand GM, Mohanty S, Devadas S. Regulators of Tfh Cell Differentiation. Front Immunol. 2016; 7: 520, doi: 10.3389/fimmu.2016.00520, indexed in Pubmed: 27933060.

37. Law H, Venturi V, Kelleher A, et al. Tfh Cells in Health and Immunity: Potential Targets for Systems Biology Approaches to Vaccination. Int J Mol Sci. 2020; 21(22), doi: 10.3390/ijms21228524, indexed in Pubmed: 33198297.

38. Veldhoen M, Uyttenhove C, van Snick I, et al. Transforming growth factor-beta ,reprograms' the differentiation of T helper 2 cells and promotes an interleukin 9-producing subset. Nat Immunol. 2008; 9(12): 1341-1346, doi: 10.1038/ni.1659, indexed in Pubmed: 18931678.

39. Van Snick J, Goethals A, Renauld JC, et al. Cloning and characterization of a cDNA for a new mouse T cell growth factor (P40). J Exp Med. 1989; 169(1): 363-368, doi: 10.1084/jem.169.1.363, indexed in Pubmed: 2521242

40. He Y, Dong L, Cao Y, et al. IL-9 and Th9 Cells in Tumor Immunity. Adv Exp Med Biol. 2020; 1240: 35-46, doi: 10.1007/978-3-030-38315-2_3, indexed in Pubmed: 32060886.

41. Rojas-Zuleta WG, Vásquez G. Th9lymphocytes: A recent history from IL-9 to its potential role in rheumatic diseases. Autoimmun Rev. 2016; 15(7): 649-655, doi: 10.1016/j.autrev.2016.02.020, indexed in Pubmed: 26921642.

42. Trifari S, Spits H. IL-22-producing CD4+ T cells: middle-men between the immune system and its environment. Eur J Immunol. 2010; 40(9): 2369-2371, doi: 10.1002/eji.201040848, indexed in Pubmed: 20809491.

43. Jia L, Wu C. The biology and functions of Th22 cells. Adv Exp Med Biol. 2014; 841: 209-230, doi: 10.1007/978-94-017-9487-9 8, indexed in Pubmed: 25261209

44. Lowes MA, Suárez-Fariñas M, Krueger JG. Immunology of psoriasis. Annu Rev Immunol. 2014; 32: 227-255, doi: 10.1146/annurev-immunol-032713-120225, indexed in Pubmed: 24655295.

45. Tian T, Yu S, Ma D. Th22 and related cytokines in inflammatory and autoimmune diseases. Expert Opin Ther Targets. 2013; 17(2): 113-125, doi: 10.1517/14728222.2013.736497, indexed in Pubmed: 23256771.

46. Chen Z, Wang Y, Ding Xi, et al. The proportion of peripheral blood Tregs among the CD4 + T cells of autoimmune thyroid disease patients: a meta-analysis. Endocr J. 2020; 67(3): 317-326, doi: 10.1507/endocrj. EJ19-0307, indexed in Pubmed: 31827051.

47. Sakaguchi S, Mikami N, Wing JB, et al. Regulatory T Cells and Human Disease. Annu Rev Immunol. 2020; 38: 541-566, doi: 10.1146/annurev-immunol-042718-041717, indexed in Pubmed: 32017635.

48. Rodríguez-Muñoz A, Vitales-Noyola M, Ramos-Levi A, et al. Levels of regulatory T cells CD69(+)NKG2D(+)IL-10(+) are increased in patients with autoimmune thyroid disorders. Endocrine. 2016; 51(3): 478-489, doi: 10.1007/s12020-015-0662-2, indexed in Pubmed: 26100786

49. O'Shea JJ, Paul WE. Mechanisms underlying lineage commitment and plasticity of helper CD4+ T cells. Science. 2010; 327(5969): 1098-1102, doi: 10.1126/science.1178334, indexed in Pubmed: 20185720.

50. Zgliczyński W. Wielka Interna. Endokrynologia. Vol II. II. Medical Tribune, Warszawa 2020.

51. Pyzik A, Grywalska E, Matyjaszek-Matuszek B, et al. Immune disorders in Hashimoto's thyroiditis: what do we know so far? J Immunol Res. 2015; 2015: 979167, doi: 10.1155/2015/979167, indexed in Pubmed: 26000316.

52. Qin Q, Liu P, Liu L, et al. The increased but non-predominant expression of Th17- and Th1-specific cytokines in Hashimoto's thyroiditis but not in Graves' disease. Braz J Med Biol Res. 2012; 45(12): 1202-1208, doi: 10.1590/s0100-879x2012007500168, indexed in Pubmed: 23090124.

53. Morshed SA, Latif R, Davies TF. Delineating the autoimmune mechanisms in Graves' disease. Immunol Res. 2012; 54(1-3): 191-203, doi: 10.1007/s12026-012-8312-8, indexed in Pubmed: 22434518.

54. Nanba T, Watanabe M, Akamizu T, et al. The -590CC genotype in the IL4 gene as a strong predictive factor for the development of hypothyroidism in Hashimoto disease. Clin Chem. 2008; 54(3): 621-623, doi: 10.1373/clinchem.2007.099739, indexed in Pubmed: 18310157.

55. Fouser LA, Wright JF, Dunussi-Joannopoulos K, et al. Th17 cytokines and their emerging roles in inflammation and autoimmunity. Immunol Rev. 2008; 226: 87-102, doi: 10.1111/j.1600-065X.2008.00712.x, indexed in Pubmed: 19161418

56. Korn T, Oukka M, Kuchroo V, et al. Th17 cells: effector T cells with inflammatory properties. Semin Immunol. 2007; 19(6): 362-371, doi: 10.1016/j. smim.2007.10.007, indexed in Pubmed: 18035554.

57. Karczewski J, Mazur M, Rychlewska-Hańczewska A, et al. [Role of Th17 lymphocytes in pathogenesis of colorectal cancer]. Postepy Hig Med Dosw (Online). 2014; 68: 42-47, doi: 10.5604/17322693.1086074, indexed in Pubmed: 24491894. 
58. Hus I, Maciag E, Roliński J. [The role of Th17 cells in anti-cancer immunity]. Postepy Hig Med Dosw (Online). 2010; 64: 244-250, indexed in Pubmed: 20498501.

59. Shi Y, Wang H, Su Z, et al. Differentiation imbalance of Th1/Th17 in peripheral blood mononuclear cells might contribute to pathogenesis of Hashimoto's thyroiditis. Scand J Immunol. 2010; 72(3): 250-255, doi: 10. 1111/j.1365-3083.2010.02425.x, indexed in Pubmed: 20696023.

60. Figueroa-Vega N, Alfonso-Pérez M, Benedicto I, et al. Increased circulating pro-inflammatory cytokines and Th17 lymphocytes in Hashimoto's thyroiditis. J Clin Endocrinol Metab. 2010; 95(2): 953-962, doi: 10.1210/jc.2009-1719, indexed in Pubmed: 20016049.

61. Zake T, Skuja S, Kalere I, et al. Upregulated tissue expression of T helper (Th) 17 pathogenic interleukin (IL)-23 and IL-1 $\beta$ in Hashimoto's thyroiditis but not in Graves' disease. Endocr J. 2019; 66(5): 423-430, doi: 10.1507/endocrj.EJ18-0396, indexed in Pubmed: 30814438.

62. Li C, Yuan J, Zhu YF, et al. Imbalance of Th17/Treg in Different Subtypes of Autoimmune Thyroid Diseases. Cell Physiol Biochem. 2016; 40(1-2): 245-252, doi: 10.1159/000452541, indexed in Pubmed: 27855396.

63. Vitales-Noyola M, Ramos-Levi AM, Martínez-Hernández R, et al. Pathogenic Th17 and Th22 cells are increased in patients with autoimmune thyroid disorders. Endocrine. 2017; 57(3): 409-417, doi: 10.1007/s12020-017-1361-y, indexed in Pubmed: 28669056.

64. Hayashi F, Watanabe M, Nanba T, et al. Association of the -31C/T functional polymorphism in the interleukin-1beta gene with the intractability of Graves' disease and the proportion of T helper type 17 cells. Clin Exp Immunol. 2009; 158(3): 281-286, doi: 10.1111/j.1365-2249.2009.04034.x, indexed in Pubmed: 19793334

65. Zheng Li, Ye P, Liu C. The role of the IL-23/IL-17 axis in the pathogenesis of Graves' disease. Endocr J. 2013; 60(5): 591-597, doi: 10.1507/endocrj. ej12-0264, indexed in Pubmed: 23327801.

66. Ciccia F, Guggino G, Rizzo A, et al. Potential involvement of IL-9 and Th9 cells in the pathogenesis of rheumatoid arthritis. Rheumatology (Oxford). 2015; 54(12): 2264-2272, doi: 10.1093/rheumatology/kev252, indexed in Pubmed: 26178600.

67. Yang Ji, Li O, Yang X, et al. Interleukin-9 Is Associated with Elevated Anti-Double-Stranded DNA Antibodies in Lupus-Prone Mice. Mol Med. 2015; 21: 364-370, doi: 10.2119/molmed.2014.00237, indexed in Pubmed: 25902303.

68. Elyaman W, Khoury SJ. Th9 cells in the pathogenesis of EAE and multiple sclerosis. Semin Immunopathol. 2017; 39(1): 79-87, doi: 10.1007/s00281-016-0604-y, indexed in Pubmed: 27844107.

69. Guggino G, Lo Pizzo M, Di Liberto D, et al. Interleukin-9 over-expression and Thelper 9 polarization in systemic sclerosis patients. Clin Exp Immunol. 2017; 190(2): 208-216, doi: 10.1111/cei.13009, indexed in Pubmed: 28681919.

70. Zivancevic-Simonovic S, Mihaljevic O, Majstorovic I, et al. Cytokine production in patients with papillary thyroid cancer and associated autoimmune Hashimoto thyroiditis. Cancer Immunol Immunother 2015; 64(8): 1011-1019, doi: 10.1007/s00262-015-1705-5, indexed in Pubmed: 25971541.

71. Chen J, Guan L, Tang L, et al. T Helper 9 Cells: A New Player in Immune-Related Diseases. DNA Cell Biol. 2019; 38(10): 1040-1047, doi: 10.1089/dna.2019.4729, indexed in Pubmed: 31414895.

72. Peng Di, Xu B, Wang Ye, et al. A high frequency of circulating th22 and th17 cells in patients with new onset graves' disease. PLoS One. 2013; 8(7): e68446, doi: 10.1371/journal.pone.0068446, indexed in Pubmed: 23874630.

73. Bai X, Sun J, Wang W, et al. Increased differentiation of Th22 cells in Hashimoto's thyroiditis. Endocr J. 2014; 61(12): 1181-1190, doi: 10.1507/endocrj.EJ14-0265, indexed in Pubmed: 25242258.

74. Vitales-Noyola M, Serrano-Somavilla A, Martínez-Hernández R, et al. Patients With Autoimmune Thyroiditis Show Diminished Levels and Defective Suppressive Function of Tr1 Regulatory Lymphocytes. J Clin Endocrinol Metab. 2018; 103(9): 3359-3367, doi: 10.1210/jc.2018-00498, indexed in Pubmed: 29982465.

75. Marazuela M, García-López MA, Figueroa-Vega N, et al. Regulatory $\mathrm{T}$ cells in human autoimmune thyroid disease. J Clin Endocrino Metab. 2006; 91(9): 3639-3646, doi: 10.1210/jc.2005-2337, indexed in Pubmed: 16804051

76. Rodríguez-Muñoz A, Vitales-Noyola M, Ramos-Levi A, et al. Levels of regulatory T cells CD69(+)NKG2D(+)IL-10(+) are increased in patients with autoimmune thyroid disorders. Endocrine. 2016; 51(3): 478-489, doi: 10.1007/s12020-015-0662-2, indexed in Pubmed: 26100786.

77. Jadidi-Niaragh F, Mirshafiey A. The deviated balance between regulatory T cell and Th17 in autoimmunity. Immunopharmacol Immunotoxicol. 2012; 34(5): 727-739, doi: 10.3109/08923973.2011.619987, indexed in Pubmed: 22316060.

78. Qin I, Zhou J, Fan $\mathrm{C}$, et al. Increased Circulating Th17 but Decreased CD4Foxp3 Treg and CD19CD1dCD5 Breg Subsets in New-Onset Graves Disease. Biomed Res Int. 2017; 2017: 8431838, doi: 10.1155/2017/8431838, indexed in Pubmed: 29259988.

79. Veldhoen M, Hocking RJ, Atkins CJ, et al. TGFbeta in the context of an inflammatory cytokine milieu supports de novo differentiation of IL-17-producing T cells. Immunity. 2006; 24(2): 179-189, doi: 10.1016/j. immuni.2006.01.001, indexed in Pubmed: 16473830.

80. Valmori D, Raffin C, Raimbaud I, et al. Human ROR $\gamma t+$ TH17 cells preferentially differentiate from naive FOXP3+Treg in the presence of lineage-specific polarizing factors. Proc Natl Acad Sci U S A. 2010; 107(45): 19402-19407, doi: 10.1073/pnas.1008247107, indexed in Pubmed: 20962281

81. Cosmi L, Maggi L, Santarlasci V, et al. T helper cells plasticity in inflammation. Cytometry A. 2014; 85(1): 36-42, doi: 10.1002/cyto.a.22348, indexed in Pubmed: 24009159. 